\title{
Audiovisual sentence recognition not predicted by susceptibility to the McGurk effect
}

\author{
Kristin J. Van Engen ${ }^{1,2,3} \cdot$ Zilong Xie $^{1} \cdot$ Bharath Chandrasekaran ${ }^{1}$
}

Published online: 5 December 2016

(C) The Psychonomic Society, Inc. 2016

\begin{abstract}
In noisy situations, visual information plays a critical role in the success of speech communication: listeners are better able to understand speech when they can see the speaker. Visual influence on auditory speech perception is also observed in the McGurk effect, in which discrepant visual information alters listeners' auditory perception of a spoken syllable. When hearing /ba/ while seeing a person saying/ga/, for example, listeners may report hearing /da/. Because these two phenomena have been assumed to arise from a common integration mechanism, the McGurk effect has often been used as a measure of audiovisual integration in speech perception. In this study, we test whether this assumed relationship exists within individual listeners. We measured participants' susceptibility to the McGurk illusion as well as their ability to identify sentences in noise across a range of signal-to-noise ratios in audio-only and audiovisual modalities. Our results do not show a relationship between listeners' McGurk susceptibility and their ability to use visual cues to understand spoken sentences in noise, suggesting that McGurk susceptibility may not be a valid measure of audiovisual integration in everyday speech processing.
\end{abstract}

Bharath Chandrasekaran

bchandra@utexas.edu

1 Department of Communication Sciences and Disorders, The University of Texas at Austin, Austin, TX, USA

2 Department of Linguistics, The University of Texas at Austin, Austin, TX, USA

3 Present address: Department of Psychological and Brain Sciences, Washington University, St. Louis, MO, USA
Keywords Audiovisual speech perception $\cdot$ Speech perception in noise $\cdot$ McGurk effect

\section{Introduction}

A brief foray into the literature on audiovisual (AV) speech perception reveals a common rhetorical approach, in which authors begin with the general claim that visual information influences speech perception and cite two effects as evidence: the McGurk effect (McGurk \& McDonald, 1976) and the intelligibility benefit garnered by listeners when they can see speakers' faces (Sumby \& Pollack, 1954). (For examples of papers that begin this way, see Altieri et al., 2011; Anderson et al., 2009; Colin et al., 2005; Grant et al., 1998; Magnotti et al., 2015; Massaro et al., 1993; Nahorna et al., 2012; Norrix et al., 2007; Ronquest et al., 2010; Rosenblum et al., 1997; Ross et al., 2007; Saalasti et al., 2011; Sams et al., 1998; Sekiyama, 1997; Sekiyama et al., 2003; Strand et al., 2014; van Wassenhove et al., 2007.) Both effects have been replicated many times and unquestionably show the influence of visual input on speech perception.

It is often assumed, then, that these two phenomena arise from a common audiovisual integration mechanism. As a result, the McGurk effect (i.e., auditory misperception of a spoken syllable when it is presented with incongruent visual information) has often been used as a measure of auditory-visual integration in speech perception. Van Wassenhove et al. (2007), for example, define AV speech integration as having occurred when "a unitary integrated percept emerges as the result of the integration of clearly differing auditory and visual informational content," and therefore use the McGurk illusion to "quantify the degree of integration that has taken place." (p. 598). Alsius et al. (2007) similarly define the degree of AV integration as "the prevalence of the McGurk effect." (p.400). 
Moreover, a number of studies investigating AV speech perception in clinical populations (e.g., individuals with schizophrenia (Pearl et al., 2009), children with amblyopia (Burgmeier et al., 2015), and individuals with Asperger syndrome (Saalasti et al., 2011)) have used the McGurk effect as their primary dependent measure of AV speech processing.

Despite the popularity of this approach, it still remains to be convincingly demonstrated that an individual's susceptibility to the McGurk illusion relates to their ability to take advantage of visual information during everyday speech processing. There is evidence that McGurk susceptibility relates (weakly) to lip-reading ability under some task and scoring conditions (Strand et al., 2014), with better lip readers being slightly more susceptible to the McGurk effect. There is also evidence linking lip-reading ability to AV speech perception (Grant et al., 1998). The connection between McGurk susceptibility and AV speech perception was directly investigated in one study on older adults with acquired hearing loss (Grant \& Seitz, 1998), with equivocal results: there was a correlation between McGurk susceptibility and visual enhancement for sentence recognition ( $r=.46)$, but McGurk susceptibility did not contribute significantly to a regression model predicting visual enhancement. The relationship between McGurk susceptibility and the use of visual information during speech perception, therefore, remains unclear. Here we present a within-subjects study of young adults with normal hearing in which we assess McGurk susceptibility and AV sentence recognition across a range of noise levels and types. If susceptibility to the McGurk effect reflects an AV integration process that is relevant to everyday speech comprehension in noise, then we expect listeners who are more susceptible to the illusion to show greater speech intelligibility gains when visual information is available for sentence recognition. If, on the other hand, different mechanisms mediate the use of auditory and visual information in the McGurk task and during everyday speech perception, then no such relationship is predicted. Such a finding would cast doubt on the utility of the McGurk task as a measure of $\mathrm{AV}$ speech perception.

\section{Method}

\section{Participants}

Thirty-nine healthy young adults $(18-29$ years; mean age = 21.03 years) were recruited from the Austin, TX, USA community. All participants were native speakers of American English and reported no history of speech, language, or hearing problems. Their hearing was screened to ensure thresholds $\leq 25 \mathrm{~dB}$ hearing level (HL) at 1,000,2,000, and $4,000 \mathrm{~Hz}$ for each ear, and their vision was normal or corrected-to-normal. Participants were compensated in accordance with a protocol approved by the University of Texas Institutional Review Board.

\section{McGurk task}

Stimuli The stimuli were identical to those in Experiment 2 of Mallick et al. (2015). They consisted of two types of AV syllables: McGurk incongruent syllables (auditory /ba/ + visual /ga/) and congruent syllables (/ba/, /da/, and /ga/). The McGurk syllables were created using video recordings from eight native English speakers (four females, four males). A different female speaker recorded the three congruent syllables. ${ }^{1}$

Procedure. The task was administered using E-Prime 2.0 software (Schneider et al., 2002). Auditory stimuli were presented binaurally at a comfortable level using Sennheiser HD280 Pro headphones, and visual stimuli were presented on a computer screen. A fixation cross was displayed for $500 \mathrm{~ms}$ prior to each stimulus. Following Mallick et al. (2015), participants were instructed to report the syllable they heard in each trial from the set /ba/, /da/, /ga/, and/tha/. The 11 stimuli (eight McGurk and three congruent) were each presented ten times. The presentation of these 110 stimuli was randomized and self-paced.

McGurk susceptibility. Responses to the McGurk incongruent stimuli were used to measure listeners' susceptibility to the McGurk effect. As in Mallick et al. (2015), responses of either /da/ or /tha/ were coded as McGurk fusion percepts.

\section{Speech perception in noise task}

Target speech stimuli A young adult male speaker of American English produced 80 simple sentences, each containing four keywords (e.g., The gray mouse ate the cheese) (Van Engen et al., 2012). Sentences were used for this task (rather than syllables) because our interest is in the relationship between McGurk susceptibility and the processing of running speech.

Maskers. Two maskers, equated for RMS amplitude, were generated to create speech-in-noise stimuli: speech-shaped noise (SSN) filtered to match the long-term average spectrum of the target speech and two-talker babble consisting of two male voices. The two maskers were included to assess listeners' ability to take advantage of visual cues in

\footnotetext{
${ }^{1}$ The unimodal intelligibility of these stimuli was tested by Mallick et al (2015). Auditory stimuli were identified with $97 \%$ accuracy $(\mathrm{SD}=4 \%)$ and visual stimuli were identified with $80 \%$ accuracy $(\mathrm{SD}=10 \%)$.
} 
different types of challenging listening environments. SSN renders portions of the target speech signal inaudible to listeners (i.e., energetic masking), while two-talker babble can also interfere with target speech identification by creating confusion and/or distraction not accounted for by the physical properties of the speech and noise (i.e., informational masking). Visual information has been shown to be more helpful to listeners when the masker is composed of other voices (Helfer \& Freyman, 2005).

Mixing targets and maskers. The audio was detached from the video recording of each sentence and equalized for RMS amplitude using Praat (Boersma \& Weenink 2010). Each audio clip was mixed with the maskers at five levels to create stimuli with the following signal-to-noise ratios (SNRs): $-4 \mathrm{~dB},-8 \mathrm{~dB},-12 \mathrm{~dB},-16 \mathrm{~dB}$, and $-20 \mathrm{~dB}$. Each noise clip was $1 \mathrm{~s}$ longer than its corresponding sentence so that $500 \mathrm{~ms}$ of noise could be played before and after each target. These mixed audio clips served as the stimuli for the audio-only (AO) condition. The audio files were also reattached to the corresponding videos to create the stimuli for the AV condition. In total, there were 400 audio (AO) files and 400 corresponding AV files with the SSN masker ( 80 sentences $\times 5$ SNRs), and 400 final audio files and 400 corresponding audiovisual files with the two-talker babble masker ( 80 sentences $\times 5$ SNRs).

Design and procedure. Masker type (SSN and two-talker babble), modality (AO and $\mathrm{AV}$ ), and $\mathrm{SNR}(-4 \mathrm{~dB},-8$ $\mathrm{dB},-12 \mathrm{~dB},-16 \mathrm{~dB}$, and $-20 \mathrm{~dB}$ ) were manipulated within subjects. Four target sentences were presented in each condition for a total of 80 trials. Trials were randomized for each participant. No sentence was repeated for a given participant.

Stimuli were presented to listeners at a comfortable level. Participants were instructed that they would be listening to $\mathrm{AO}$ and $\mathrm{AV}$ sentences in noise, and were told that the target sentences would begin a half second after the noise. The participant initiated each stimulus presentation using the keyboard, and they were given unlimited time to respond. If they were unable to understand a sentence, they were asked to report any intelligible words and/or make their best guess. If they did not understand anything, they were told to type 'X.' For AO trials, a centered crosshair was presented on the screen during the audio stimulus; for AV trials, a full-screen video of the speaker was presented with the audio. Responses were scored by the number of keywords identified correctly. Homophones and obvious spelling errors were scored as correct; words with added or deleted morphemes were scored as incorrect.

\section{Results}

\section{McGurk susceptibility}

Figure 1 shows the distribution of McGurk susceptibility scores. As in Mallick et al. (2015), scores ranged from $0 \%$ to $100 \%$ and were skewed to the right.

\section{Keyword identification in noise}

Average keyword intelligibility across SNRs is shown in Fig. 2. The full set of identification data was first analyzed to determine whether McGurk susceptibility predicted keyword identification under any of the test conditions. Statistical analysis was performed using the lme4 package (version 1.1-12, Bates et al., 2015) for R software (2016). The response to each keyword was categorized as correct or incorrect and analyzed using a mixed logit model for binomially distributed outcomes. Because comparing the noise types to one another was not of primary interest, separate analyses were conducted for the two noise types to simplify model interpretation. For each analysis, modality, SNR, McGurk scores, and their 2- and 3-way interactions were entered as fixed factors. Modality was deviation-coded (i.e., -0.5 and 0.5 ), which entails that the coefficient represents modality's "main effect" (i.e., its partial effect when all others are zero). Continuous predictors (SNR and McGurk scores) were centered and scaled. The models were fit with the maximal random effects structure justified by the experimental design (Barr et al., 2013). ${ }^{2}$ The model outputs for the fixed effects are shown in Table 1 and Table 2.

For both types of noise, modality, SNR, and their interaction were the only statistically significant predictors of keyword identification in noise. Neither McGurk susceptibility nor its interactions with SNR or modality significantly predicted keyword identification. That said, McGurk scores and their interaction with modality did near statistical significance ( $p=.10, p=.07)$ in SSN. As shown in Fig. 3, these trends reflect a negative association between McGurk susceptibility, particularly in the AV conditions.

\section{Visual enhancement}

One way researchers quantify individuals' ability to use visual information during speech identification tasks is by calculating visual enhancement (VE), which takes the difference between a listener's performance in $\mathrm{AV}$ and $\mathrm{AO}$ conditions and normalizes it by the proportion of improvement available

\footnotetext{
${ }^{2}$ For this study, the maximal random effects structure included random intercepts for subjects and sentences and random slopes for the following: subject by modality, subject by SNR, subject by the interaction of modality and SNR, sentence by modality, sentence by SNR, and sentence by the interaction of modality and SNR.
} 
Distribution of McGurk susceptibiliy scores

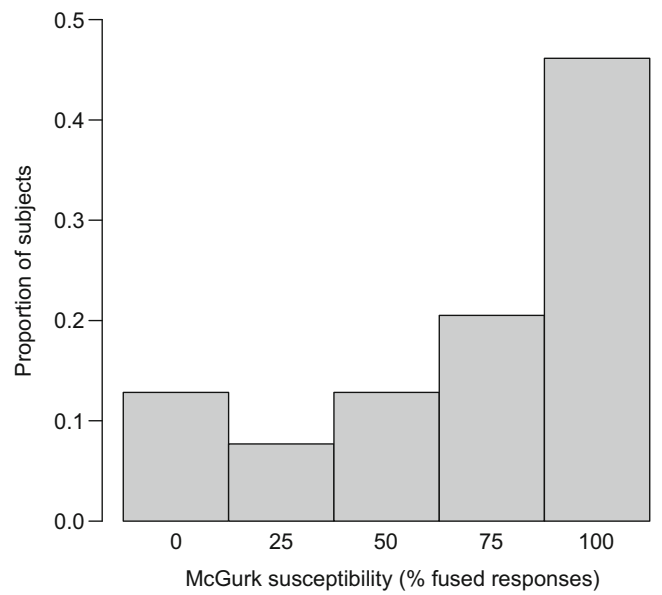

Fig. 1 Distribution of McGurk susceptibility scores

given their AO performance (Grant \& Seitz, 1998; Grant et al., 1998; Sommers et al., 2005):

$\mathrm{VE}=\frac{(\mathrm{AV}-\mathrm{AO})}{(1-\mathrm{AO})}$

Because AO performance must be below ceiling to calculate VE (i.e., there must be room for improvement), VE was calculated for the SNRs of $-8 \mathrm{~dB}$ and below. (At least $10 \%$ of the subjects were at ceiling in $\mathrm{AO}$ at $-4 \mathrm{~dB}$ ). For each listener, VE was calculated separately for two-talker babble and SSN. A positive VE score indicates that a listener identified more words in the AV condition than in the AO condition; the maximum VE score of 1 indicates that the listener identified all of the keywords in the AV condition.

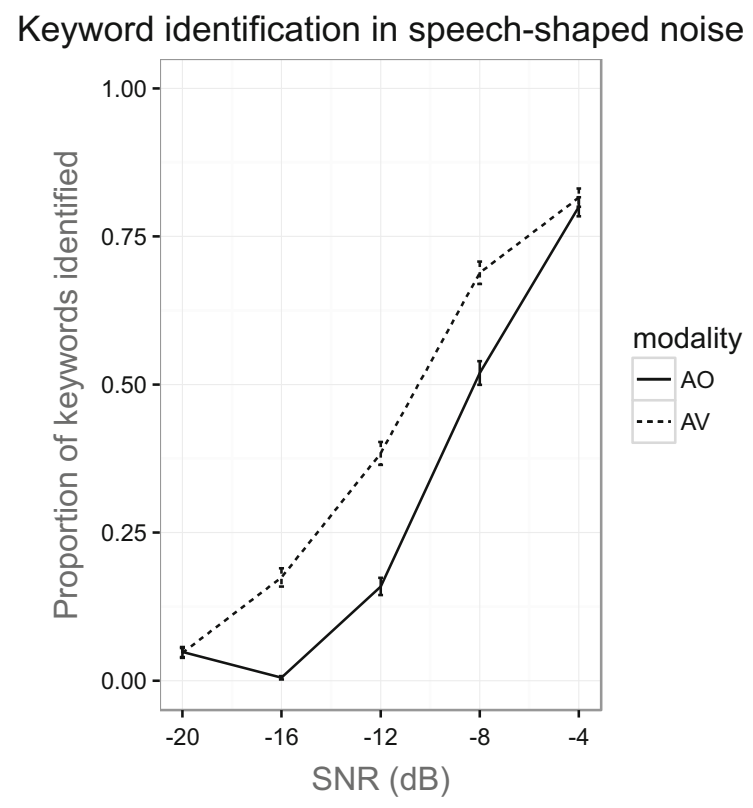

Table 1 Speech-shaped noise

\begin{tabular}{lllll}
\hline Fixed effects & Estimate & SE & Z value & P-value \\
\hline Intercept & -1.41497 & 0.20652 & -6.851 & $<0.001$ \\
Modality & 1.58603 & 0.27298 & 5.810 & $<0.001$ \\
SNR & -2.78651 & 0.15256 & -18.266 & $<0.001$ \\
McGurk & -0.29178 & 0.17577 & -1.660 & 0.09690 \\
Modality $\times$ SNR & 0.79314 & 0.30170 & 2.629 & 0.00857 \\
Modality $\times$ McGurk & -0.37224 & 0.20761 & -1.793 & 0.07297 \\
SNR $\times$ McGurk & 0.03589 & 0.10334 & 0.347 & 0.72836 \\
Modality $\times$ SNR $\times$ McGurk & 0.21852 & 0.19936 & 1.096 & 0.27303 \\
\hline
\end{tabular}

Results are shown in Fig. 4. One outlier is not displayed because it would have required a significant extension of the y-axis. (In SSN at $-8 \mathrm{~dB}$, the individual identified $\sim 94 \%$ of $\mathrm{AO}$ words, but only $50 \%$ of the AV words, resulting in a VE score of -7.) All other data are shown. Although it is not the focus of this study, it is worth noting that the VE data do not follow the principle of inverse effectiveness (Holmes, 2009), which predicts greater multisensory integration in more difficult conditions. If anything, the data for SSN suggest the opposite pattern: greater VE at easier SNRs. (See also Van Engen et al. 2014, Tye-Murray et al., 2010, and Ross et al., 2007 for other cases where this principle does not capture behavior in AV speech perception.)

Figure 5 displays the data for each condition with McGurk susceptibility scores plotted against VE scores. Due to the skewness of the McGurk data (see Fig. 1), Kendall's tau was used to assess the relationship between individuals' rate of McGurk responses and their VE scores. As shown in Table 3, there was no statistically significant

\section{Keyword identification in 2-talker babble}

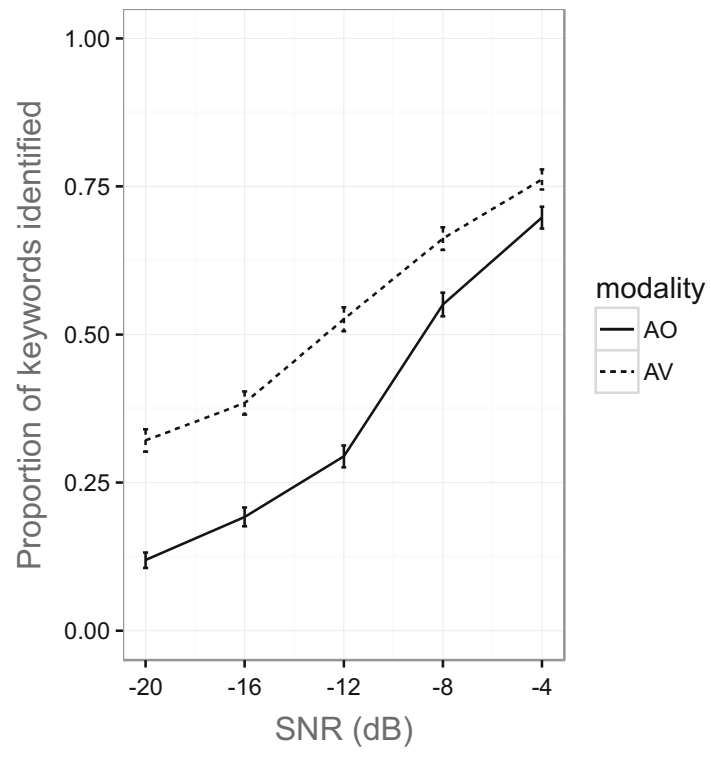

Fig. 2 Proportion of keywords identified across SNRs in speech-shaped noise (left) and 2-talker babble (right). Error bars represent standard error 
Table 2 Two-talker babble

\begin{tabular}{lllll}
\hline Fixed effects & Estimate & SE & Z value & P-value \\
\hline Intercept & -0.67487 & 0.28097 & -2.402 & 0.0163 \\
Modality & 1.37872 & 0.20354 & 6.774 & $<0.001$ \\
SNR & -1.56604 & 0.12803 & -12.231 & $<0.001$ \\
McGurk & -0.21197 & 0.25670 & -0.826 & 0.4090 \\
Modality $\times$ SNR & 0.80622 & 0.17795 & 4.531 & $<0.001$ \\
Modality $\times$ McGurk & -0.15580 & 0.15740 & -0.990 & 0.3223 \\
SNR $\times$ McGurk & 0.06916 & 0.09763 & 0.708 & 0.4787 \\
Modality $\times$ SNR $\times$ McGurk & -0.16874 & 0.10971 & -1.538 & 0.1240 \\
\hline
\end{tabular}

association in any condition (p-values ranged from .06 to .85 ; tau values ranged from -.21 to .06 ).

In SSN at $-8 \mathrm{~dB}$ and in two-talker babble at $-16 \mathrm{~dB}$, this correlation neared statistical significance $(p=.190$ and $p=.063)$ with negative tau values, indicating that the (possible) relationship between VE and McGurk susceptibility is one in which susceptibility to the illusion is associated with less VE. In both the analysis of keyword identification and VE, then, no association between McGurk susceptibility and speech perception in noise was significant at the $\mathrm{p}<.05$ level, and the two marginally significant relationships suggested that susceptibility to the illusion was associated with lower rates of keyword identification and less visual enhancement.

\section{Discussion}

The results of this experiment showed no statistically significant relationship between an individual's susceptibility to the McGurk effect and their ability to understand speech in noise (with or without visual information). Importantly, the VE analyses also showed no significant relationship between an individual's susceptibility to the McGurk illusion and their ability to use visual cues in order to improve on audio-only speech perception. ${ }^{3}$ Research that is fundamentally interested in understanding audiovisual integration as it relates to a listener's ability to understand connected speech, therefore, should not assume that susceptibility to the McGurk effect can be used as a valid measure of AV speech processing.

\footnotetext{
${ }^{3}$ This result contrasts with that of Grant and Seitz (1998), which showed a significant correlation between McGurk susceptibility and visual enhancement for sentence recognition in older adults with hearing loss (note, however, that the regression analysis in that study also indicated McGurk susceptibility was not a significant predictor of VE). Given the different stimulus materials, noise levels, and listener populations in the two studies, there are multiple possible explanations for the different outcomes. Differences in unimodal abilities across the participants in the two studies are a likely candidate: not only did the older adults in Grant and Seitz (1998) have hearing loss, but speechreading ability is also known to decline with age (Sommers et al., 2005; Tye-Murray et al., 2016).
}

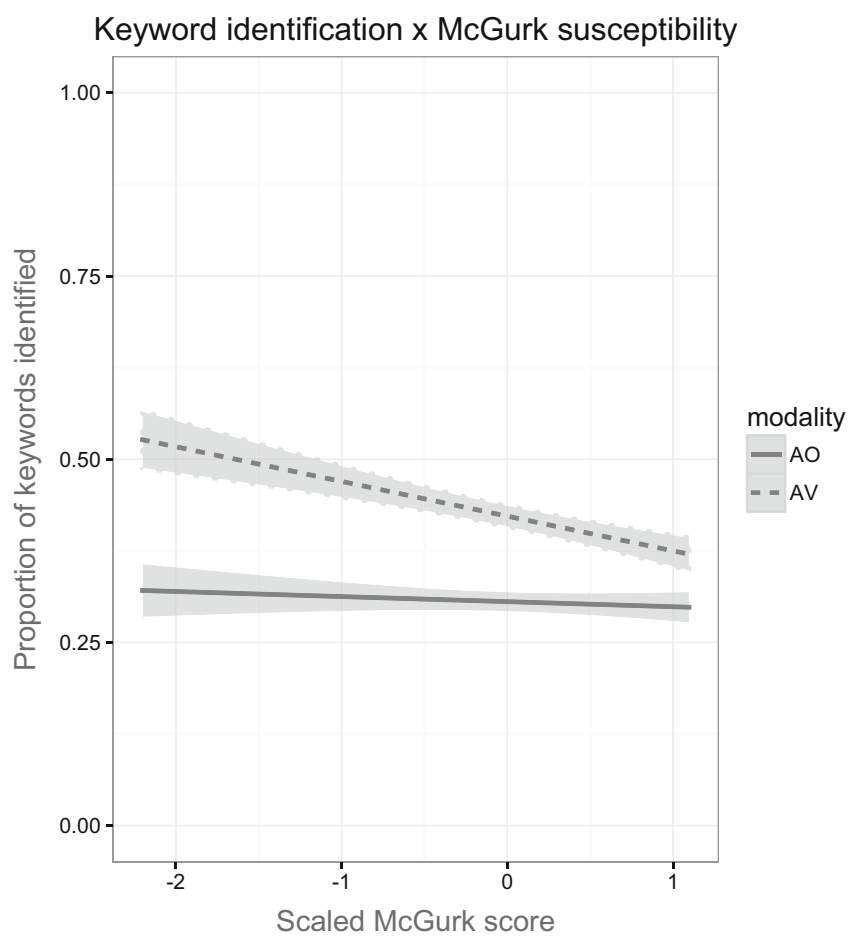

Fig. 3 Relationship between McGurk susceptibility scores (scaled) and proportion of keywords identified in speech-shaped noise

Although the McGurk effect and the benefit of visual information both show that visual information affects auditory processing, there are several critical differences between these phenomena. One noteworthy difference is the congruency of the auditory and visual information in these situations. In the McGurk effect, auditory identification of individual consonants is altered by conflicting visual information; visual information can be thought of, therefore, as detrimental to correct auditory perception. In AO versus AV speech in noise, congruent visual information facilitates auditory perception. At least one recent neuroimaging study supports the hypothesis that different neural mechanisms may mediate the integration of congruent versus incongruent visual information with auditory signals. Using functional magnetic resonance imaging (fMRI), Erickson et al. (2014) showed that distinct posterior superior temporal regions are involved in processing congruent $\mathrm{AV}$ speech and incongruent $\mathrm{AV}$ speech when compared to unimodal speech (acoustic-only and visual-only). Left posterior superior temporal sulcus (pSTS) was recruited during congruent bimodal AV speech. In contrast, left posterior superior temporal gyrus (pSTG) was recruited when processing McGurk speech, suggesting that left pSTG may be necessary when there is a discrepancy between auditory and visual cues. It may be that left pSTG is involved in the generation of the fused percept that can arise from conflicting cues.

Another critical difference between McGurk tasks and sentence-in-noise tasks is the amount of linguistic context available to listeners. The top-down and bottom-up processes 


\section{VE scores by condition}

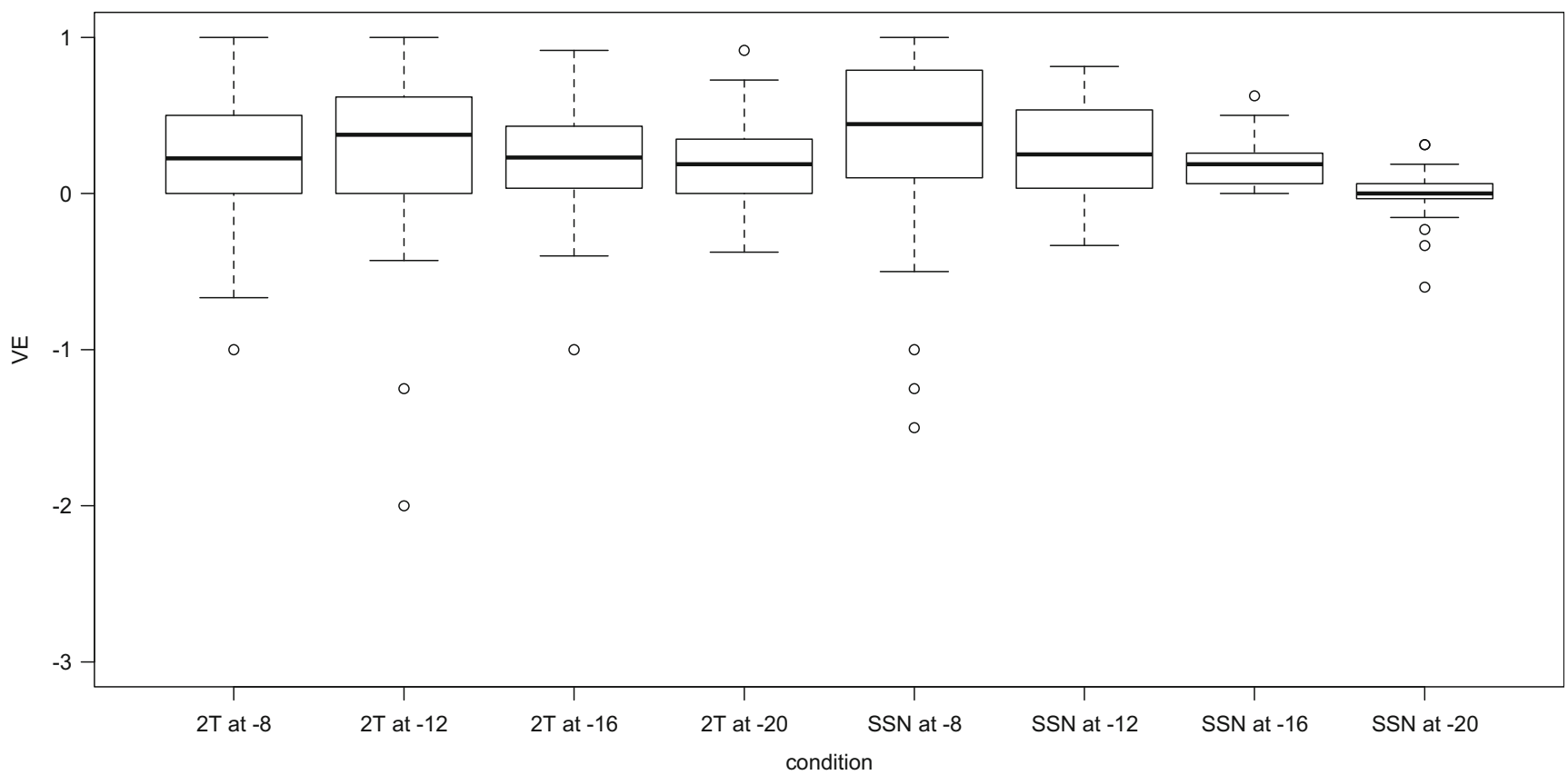

Fig. 4 Visual enhancement (VE) scores by condition. The boxes extend from the 25 th percentile to the 75 th percentile, with the dark line indicating the median. Whiskers extend to data points that are within

involved in identifying speech vary significantly with listeners' access to lexical, syntactic, and semantic context (Mattys et al., 2005), and the availability of rhythmic information in running speech allows for neural entrainment and
1.5 times the interquartile range. Data outside that range are denoted by open circles. Note that one outlier is not displayed (a VE score of -7 on $\mathrm{SSN}$ at $-8 \mathrm{~dB}$ )

predictive processing that is not possible when identifying isolated syllables (Peelle \& Davis, 2012; Peelle \& Sommers, 2015). In keeping with these observations, previous studies have failed to show a relationship between $\mathrm{AV}$ integration
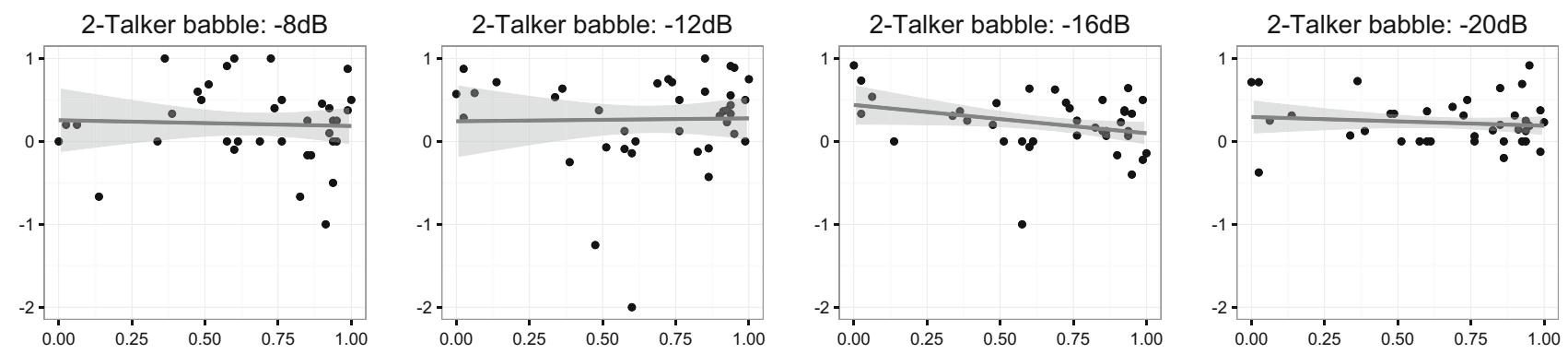

щ
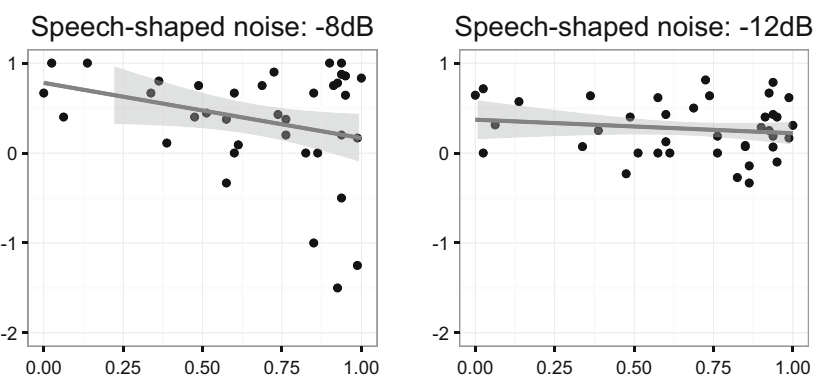

Speech-shaped noise: $-16 \mathrm{~dB}$
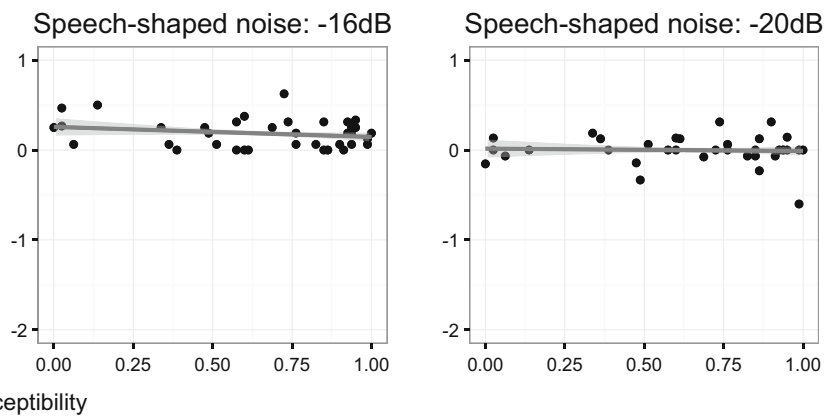

Fig. 5 Visual enhancement plotted against McGurk susceptibility for each listening condition. Top row: Two-talker babble conditions. Bottom row: Speech-shaped noise conditions. Linear trendlines are

consistency, one data point for $\mathrm{SSN}-8 \mathrm{~dB}$ is not displayed, although it is included in the calculation of the trendline (McGurk score=.86; VE score $=-7)$ 
Table 3 Results of correlation tests (Kendall's tau) for the relationship between McGurk susceptibility and visual enhancement (VE)

\begin{tabular}{llll}
\hline & $\mathrm{z}$ & $\mathrm{p}$-value & tau \\
\hline $\mathrm{SSN}:-8 \mathrm{~dB}$ & -1.312 & 0.190 & -0.150 \\
$\mathrm{SSN}:-12 \mathrm{~dB}$ & -0.376 & 0.707 & -0.043 \\
$\mathrm{SSN}:-16 \mathrm{~dB}$ & -.676 & 0.499 & -0.080 \\
$\mathrm{SSN}:-20 \mathrm{~dB}$ & -0.192 & 0.848 & -0.023 \\
$2 \mathrm{~T}:-8 \mathrm{~dB}$ & -0.261 & 0.794 & -0.031 \\
$2 \mathrm{~T}:-12 \mathrm{~dB}$ & 0.533 & 0.594 & 0.060 \\
$2 \mathrm{~T}:-16 \mathrm{~dB}$ & -1.855 & 0.063 & -0.210 \\
$2 \mathrm{~T}:-20 \mathrm{~dB}$ & -0.612 & 0.541 & -0.071 \\
\hline
\end{tabular}

measures derived from consonant versus sentence recognition (Grant \& Seitz, 1998; Sommers et al., 2005). Grant and Seitz (1998) showed no correlation between the two in older adults with hearing impairment, and Sommers et al. (2005) found that, while VE for word and sentence identification were related to one another for young adults, VE for consonant identification was not related to either. Given that other researchers have shown significant relationships between consonant identification and words or sentences in unimodal conditions (Grant et al., 1998; Humes et al., 1994; Sommers et al. 2005), these results suggest that the lack of relationship between VE for consonants and VE for words and sentences results from differences in the mechanisms mediating the integration of auditory and visual inputs for these different types of speech materials.

The results reported here serve two purposes: First, as a caution against the assumption that the McGurk effect can be used as an assay of audiovisual integration for speech in challenging listening conditions; and second, to set the scene for future work investigating the potentially different mechanisms supporting the integration of auditory and visual information across different types of speech materials.

Acknowledgments This work was supported by NIH-NIDCD grant R01DC013315 to BC. We thank the SoundBrain laboratory research assistants, especially Jacie Richardson and Cat Han, for data collection and scoring. The content is solely the responsibility of the authors and does not necessarily represent the official views of the National Institutes of Health.

\section{References}

Altieri, N., Pisoni, D. B., \& Townsend, J. T. (2011). Some behavioral and neurobiological constraints on theories of audiovisual speech integration: a review and suggestions for new directions. Seeing and Perceiving, 24(6), 513-539. doi:10.1163/187847611X595864

Alsius, A., Navarra, J., \& Soto-Faraco, S. (2007). Attention to touch weakens audiovisual speech integration. Experimental Brain Research, 183(3), 399-404. doi:10.1007/s00221-007-1110-1

Andersen, T. S., Tiippana, K., Laarni, J., Kojo, I., \& Sams, M. (2009). The role of visual spatial attention in audiovisual speech perception.
Speech Communication, 51(2), 184-193. doi:10.1016/j. specom.2008.07.004

Barr, D. J., Levy, R., Scheepers, C., \& Tily, H. J. (2013). Random effects structure for confirmatory hypothesis testing: Keep it maximal. Journal of Memory and Language, 68(3), 255-278. doi:10.1016/j. jml.2012.11.001

Bates, D., Mächler, M., Bolker, B. M., \& Walker, S. C. (2015). Fitting Linear Mixed- Effects Models using lme4. Journal of Statistical Software, 67(1), 1-48. doi:10.18637/jss.v067.i01

Boersma, P., \& Weenink, D. (2010). Praat: doing phonetics by computer (Version 5.1). Retrieved from http://www.praat.org

Burgermeier, R., Desai, R. U., Farner, K. C., Tiano, B., Lacey, R., Volpe, N. J., \& Mets, M. B. (2015). The Effect of Amblyopia on VisualAuditory Speech Perception, 133(1), 11.doi:10.1001 /jamaophthalmol.2014.3307

Colin, C., Radeau, M., \& Deltenre, P. (2005). Top-down and bottom-up modulation of audiovisual integration in speech. European Journal of Cognitive Psychology, 17(4), 541-560. doi:10.1080 109541440440000168

Erickson, L. C., Zielinski, B. A., Zielinski, J. E. V., Liu, G., Turkeltaub, P. E., Leaver, A. M., \& Rauschecker, J. P. (2014). Distinct cortical locations for integration of audiovisual speech and the McGurk effect. Frontiers in Psychology, 5(158), 265. doi:10.3389 /fpsyg.2014.00534

Grant, K. W., Walden, B. E., \& Seitz, P. F. (1998). Auditory-visual speech recognition by hearing-impaired subjects: consonant recognition, sentence recognition, and auditory-visual integration. The Journal of the Acoustical Society of America, 103(5), 2677-2690. doi: $10.1121 / 1.422788$

Grant, K. W., \& Seitz, P. F. (1998). Measures of auditory-visual integration in nonsense syllables and sentences. The Journal of the Acoustical Society of America, 104(4), 2438-2450. doi:10.1121 /1.423751

Helfer, K. S., \& Freyman, R. L. (2005). The role of visual speech cues in reducing energetic and informational masking. The Journal of the Acoustical Society of America, 117(2), 842-849. doi:10.1121 $/ 1.1836832$

Holmes, N. P. (2009). The Principle of Inverse Effectiveness in Multisensory Integration: Some Statistical Considerations. Brain Topography, 21(3-4), 168-176. doi:10.1007/s10548-009-0097-2

Humes, L. E., Watson, B. U., Christensen, L. A., Cokely, C. G., Halling, D. C., \& Lee, L. (1994). Factors associated with individual differences in clinical measures of speech recognition among the elderly. Journal of Speech Language and Hearing Research, 37(2), 465474. doi:10.1044/jshr.3702.465

Mallick, D. B., Magnotti, J. F., \& Beauchamp, M. S. (2015). Variability and stability in the McGurk effect: contributions of participants, stimuli, time, and response type. Psychonomic Bulletin \& Review, 22(5), 1299-1307. doi:10.3758/s13423-015-0817-4

McGurk, H., \& MacDonald, J. (1976). Hearing lips and seeing voices. Nature, 264(5588), 746-748. doi:10.1038/264746a0

Magnotti, J. F., Mallick, D. B., Feng, G., Zhou, B., Zhou, W., \& Beauchamp, M. S. (2015). Similar frequency of the McGurk effect in large samples of native Mandarin Chinese and American English speakers. Experimental Brain Research, 233(9), 2581-2586. doi:10.1007/s00221-015-4324-7

Massaro, D. W., Cohen, M. M., Gesi, A., Heredia, R., \& Tsuzaki, M. (1993). Bimodal speech perception: an examination across languages. Journal of Phonetics, 21, 445-478.

Mattys, S. L., White, L., \& Melhorn, J. F. (2005). Integration of Multiple Speech Segmentation Cues: A Hierarchical Framework. Journal of Experimental Psychology: Learning, Memory, and Cognition, 134(4), 477-500. doi:10.1037/0096-3445.134.4.477

Nahorna, O., \& Berthommier, F. (2012). Binding and unbinding the auditory and visual streams in the McGurk effect. The Journal of the Acoustical Society of America, 1061-1077. doi:10.1121/1.4728187 
Norrix, L. W., Plante, E., Vance, R., \& Boliek, C. A. (2007). AuditoryVisual Integration for Speech by Children With and Without Specific Language Impairment. Journal of Speech Language and Hearing Research, 50(6), 1639-1651. doi:10.1044/1092-4388 $(2007 / 111)$

Pearl, D., Yodashkin-Porat, D., Katz, N., Valevski, A., Aizenberg, D., Sigler, M., ... Kikinzon, L. (2009). Differences in audiovisual integration, as measured by McGurk phenomenon, among adult and adolescent patients with schizophrenia and age-matched healthy control groups. Comprehensive Psychiatry, 50(2), 186-192. doi:10.1016/j.comppsych.2008.06.004

Peelle, J. E., \& Davis, M. H. (2012). Neural oscillations carry speech rhythm through to comprehension. Frontiers in Psychology, 3. doi:10.3389/fpsyg.2012.00320

Peelle, J. E., \& Sommers, M. S. (2015). Prediction and constraint in audiovisual speech perception. Cortex, 68, 169-181. doi:10.1016 /j.cortex.2015.03.006

R Core Team (2016). R: A language and environment for statistical computing. R Foundation for Statistical Computing, Vienna, Austria. URL https://www.R-project.org

Ronquest, R. E., Levi, S. V., \& Pisoni, D. B. (2010). Language identification from visual-only speech signals. Attention, Perception \& Psychophysics, 72(6), 1601-1613. doi:10.3758/app.72.6.1601

Rosenblum, L. D., Schmuckler, M. A., \& Johnson, J. A. (1997). The McGurk effect in infants. Perception and Psychophysics, 59(3), 347-357. doi:10.3758/BF03211902

Ross, L. A., Saint-Amour, D., Leavitt, V. M., Javitt, D. C., \& Foxe, J. J. (2007). Do you see what I am saying? Exploring visual enhancement of speech comprehension in noisy environments. Cerebral Cortex, 17(5), 1147-1153. doi:10.1093/cercor/bhl024

Saalasti, S., Kätsyri, J., Tiippana, K., Laine-Hernandez, M., von Wendt, L., \& Sams, M. (2011). Audiovisual Speech Perception and Eye Gaze Behavior of Adults with Asperger Syndrome. Journal of Autism and Developmental Disorders, 42(8), 1606-1615. doi:10.1007/s10803-011-1400-0

Sams, M., Manninen, P., Surakka, V., \& Helin, P. (1998). McGurk effect in Finnish syllables, isolated words, and words in sentences: Effects of word meaning and sentence context. Speech Communication, 26(1-2), 75-87. doi:10.1016/s0167-6393(98)00051-x
Schneider, W., Eschman, A., \& Zuccolotto, A. (2002). E-Prime User's Guide. Pittsburgh: Psychology Software Tools, Inc.

Sekiyama, K. (1997). Cultural and linguistic factors in audiovisual speech processing: The McGurk effect in Chinese subjects. Perception and Psychophysics, 59(1), 73-80. doi:10.3758/BF03206849

Sekiyama, K., Kanno, I., Miura, S., \& Sugita, Y. (2003). Auditory-visual speech perception examined by fMRI and PET. Neuroscience Research, 47(3), 277-287. doi:10.1016/S0168-0102(03)00214-1

Sommers, M. S., Tye-Murray, N., \& Spehar, B. (2005). Auditory-visual speech perception and auditory-visual enhancement in normalhearing younger and older adults. Ear and Hearing, 26(3), 263275. doi:10.1097/00003446-200506000-00003

Strand, J., Cooperman, A., Rowe, J., \& Simenstad, A. (2014). Individual differences in susceptibility to the McGurk effect: links with lipreading and detecting audiovisual incongruity. Journal of Speech Language and Hearing Research, 57(6), 2322-2331. doi:10.1044/2014_JSLHR-H-14-0059

Sumby, W. H., \& Pollack, I. (1954). Visual contribution to speech intelligibility in noise. The Journal of the Acoustical Society of America, 26(2), 212-215. doi:10.1121/1.1907309

Tye-Murray, N., Sommers, M., Spehar, B., \& Myerson, J. (2010). Aging, audiovisual integration, and the principle of inverse effectiveness. Ear and Hearing, 1.doi:10.1097/aud.0b013e3181ddf7ff

Tye-Murray, N., Spehar, B., Myerson, J., Hale, S., \& Sommers, M. (2016). Lipreading and audiovisual speech recognition across the adult lifespan: implications for audiovisual integration. Psychology and Aging, 31(4), 380-389. doi:10.1037/pag0000094

Van Engen, K. J., Chandrasekaran, B., \& Smiljanic, R. (2012). Effects of speech clarity on recognition memory for spoken sentences. PloS One, 7(9), e43753. doi:10.1371/journal.pone.0043753

Van Engen, K. J., Phelps, J. E. B., Smiljanic, R., \& Chandrasekaran, B. (2014). Enhancing speech intelligibility: interactions among context, modality, speech style, and masker. Journal of Speech, Language, and Hearing Research 57, 1908-1918. doi:10.1044 /JSLHR-H-13-0076

van Wassenhove, V., Grant, K. W., \& Poeppel, D. (2007). Temporal window of integration in auditory-visual speech perception. Neuropsychologia, 45(3), 598-607. doi:10.1016/j. neuropsychologia.2006.01.001 